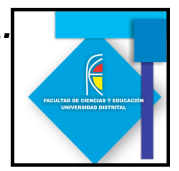

\title{
PROPUESTA ETNOEDUCATIVA: LA FORMACIÓN DEL MUNDO DESDE DOS PERSPECTIVAS DIFERENTES
}

\section{AN ETHICAL EDUCATIONAL PROPOSAL: THE ORIGIN OF THE WORLD FROM TWO DIFFERENT PERSPECTIVES}

\author{
Ronal Enrique Callejas Arévalo ${ }^{1}$ \\ Yeni Milena Rueda Velasco ${ }^{2}$ \\ Franceidy Andrea Salazar Garzón ${ }^{3}$
}

\section{Resumen}

La Constitución Colombiana es clara afirmando que la educación es un servicio y un derecho que debe ser impartida a todas las culturas por igual, reconociendo el carácter pluriétnico y multicultural del país. Con base en esta aseveración el presente trabajo tiene como objetivo reconocer la cosmovisión de los integrantes del Cabildo Muisca de Bosa acerca de la formación del mundo, contrastándola frente a la representación física de este evento. Para tal reconocimiento de saberes, se plantea una estrategia didáctica que de manera respetuosa y atenta muestra la cosmovisión del pueblo muisca y la representación física de la formación del mundo. La estrategia didáctica presenta un cuento interactivo, dirigido a niños integrantes del cabildo muisca cuyas edades oscilan entre los 7 y 9 años. La estrategia didáctica, consta de tres fases reflejadas en el cuento, las cuales llevan por nombre: ¿Qué tanto sabemos?, reconozcamos dos saberes y reflexionemos; cada una de estas fases cuenta con un grupo de actividades de interacción con el lector, buscando generar una actitud activa durante la ejecución de la estrategia.

Palabras clave: Etnoeducación, cuento interactivo, Ley de Origen, Big Bang.

\begin{abstract}
The Colombian Constitution is clear in stating that education is a service and a right that should be given to every people independent of his ethnic origin, recognizing the multiethnic and multicultural character of our country. Thus this work is aimed to recognize the worldview that members of the "Cabildo Muisca" at Bosa, Colombia, have about the Word's origin and compare it with the one proposed by the physicists. To recognize that knowledge, we propose a didactical strategy that respectfully and cordially shows both, the muisca's worldview and the physicists' representation of the word's origin.
\end{abstract}

\footnotetext{
${ }_{1}^{1}$ Profesor de la Universidad Pedagógica Nacional, rcallare@hotmail.com

${ }^{2}$ Estudiante de licenciatura en física de la Universidad Pedagógica Nacional, yeni_milenar@hotmail.com

${ }^{3}$ Estudiante de licenciatura en física de la Universidad Pedagógica Nacional, fransagar_1416@hotmail.com
} 


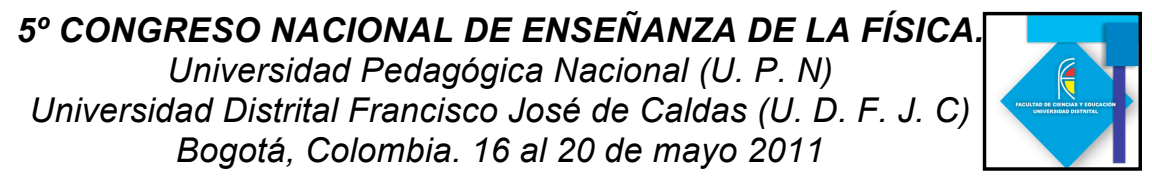

In this didactical strategy an interactive story is presented to muiscas's children, whose ages are from 7 to 9 . This strategy consists of three major phases which are reflected in the story, entitled, How much do we know? Let us recognize two types of knowledge and Let us reflect. In each of these phases there is a group of activities for the reader to interact with the story aimed to generate an active attitude while the strategy is being carried out.

Keywords: Ethno-education, interactive story, Original Law, Big Bang.

\section{Introducción}

Colombia cuenta con una riqueza cultural que requiere de una atención individual y respetuosa, si no se tienen en cuenta las particularidades que distinguen a cada cultura, éstas podrían verse envueltas en un proceso de aculturalización, que podría provocar la pérdida del carácter pluriétnico del país. Para mitigar este proceso, es preciso formar al sujeto como ser social capaz de reconocer y validar otras culturas sin olvidar o rechazar la propia. Al ser la escuela la encargada de formar al individuo como ser social exige una preparación adecuada del docente, que le permita desempeñar con solvencia su labor en los diferentes ambientes del aprendizaje y a su vez pueda aportar al desarrollo de competencias sociales y culturales en los integrantes de cada una de estas culturas.

Uno de los escenarios donde se hacen evidentes estas necesidades es el Cabildo Muisca de Bosa, es por esto, que el acercamiento con el cabildo busca caracterizar y validar la cosmovisión muisca, para así propiciar una enseñanza basada en las costumbres y creencias de esta cultura. Como estrategia didáctica se ha diseñado un cuento interactivo que tiene como intención acercar al lector a la formación del mundo visto desde dos perspectivas diferentes, la brindada por la comunidad muisca y la establecida por la comunidad científica.

\section{El cuento en la enseñanza}

Desde que se empezó a reconocer a la literatura como herramienta que permite despertar el interés del sujeto hacia la lectura, se han diseñado numerosos textos, entre ellos los narrativos, dentro los cuales se destaca la narrativa a través del cuento, el cual genera en el lector: el asombro, la creatividad, el ingenio y el interés por encontrar algo nuevo que estimule su imaginación.

Cuando el sujeto tiene la autonomía de diseñar, crear o participar en un cuento, puede apropiarse del contenido, cuestionándose y dando lugar a una lluvia de ideas que más adelante podrían servir como respuesta a sus cuestionamientos.

\section{Estrategia didáctica: Las aventuras de Yopo en dos mundos}

Cómo estrategia didáctica se diseñó un cuento interactivo donde los niños guiado por el maestro puede participar activamente, estimulando un aprendizaje critico acerca del mito y creencias de la cultura muisca sobre la «Ley de Origen» y a su vez aproxime al lector a 


\section{$5^{\circ}$ CONGRESO NACIONAL DE ENSEÑANZA DE LA FÍSICA. \\ Universidad Pedagógica Nacional (U. P. N) \\ Universidad Distrital Francisco José de Caldas (U. D. F. J. C) \\ Bogotá, Colombia. 16 al 20 de mayo 2011

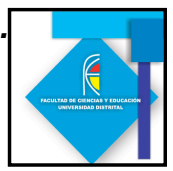

la explicación física de éste evento. La estrategia didáctica está

compuesta por tres fases, cada una de las cuales tiene un propósito particular.

\section{1. ¿Qué tanto sabemos?}

El propósito de esta fase es recoger los imaginarios que los niños tienen respecto a la formación del mundo, para ello se diseñaron diferentes actividades, donde se estimula la creatividad e imaginación del lector, con un acompañamiento constante del maestro se pretende guiar al estudiante a plasmar dichas habilidades por medio de dibujo, la escritura y el dialogo en esta primera parte.

\section{Reconozcamos dos saberes}

En esta segunda fase, se quiere aproximar al lector a dos de las tantas representaciones existentes sobre la formación del mundo. Las dos perspectivas mostradas en el cuento son, la cosmovisión brindada por la cultura muisca y la representación física aceptada en la cultura occidental. Se proponen una variedad de actividades cuyo propósito principal es hacer partícipe e involucrar al estudiante en la trama del cuento y a su vez le permita suscitar cuestionamientos en el momento en que las dos perspectivas intentan ser contrastadas.

\section{Reflexionemos}

A esta fase los niños llegan con los cuestionamientos adquiridos en la fase anterior, por tanto, ésta tiene la gran responsabilidad de esclarecer las dudas, al validar las dos perspectivas desde una mirada intercultural.

Las actividades propuestas en esta fase permiten evaluar si ellos reconocen las dos representaciones narradas en el cuento y si tal vez las validan.

\section{Personajes principales del cuento:}

Yopo, quien gracias a su gran imaginación es el encargado de ilustrar las diferentes teorías al lector, la maestra Anny y el abuelo Chipo, son quienes narran la representación física y la cosmología muisca, respectivamente.

\section{Abordajes del cuento}

\section{Cosmología muisca}

Los muiscas al tener una cultura propia, poseen una cosmovisión autóctona acerca de la formación del mundo o Ley de Origen, nombre dado por ellos, la cual plantea al hombre como centro del mundo y encargado de mantener vivo el amor por la madre tierra, según su cosmovisión antes que nada o primero que todo sólo existía la oscuridad y el pensamiento de la Madre Abuela Bague. Quien gracias a su gran sabiduría, brindo a sus hijos o dioses las herramientas que ellos utilizaron para dar vida a parte de su pensamiento. Fue así que la primera tarea desempeñada por los dioses fue crear las direcciones para poder ubicarse, misteriosamente se dieron cuenta que todas las direcciones coincidían en un lugar mágico al que llamaron tomsa, el ombligo, al darse 


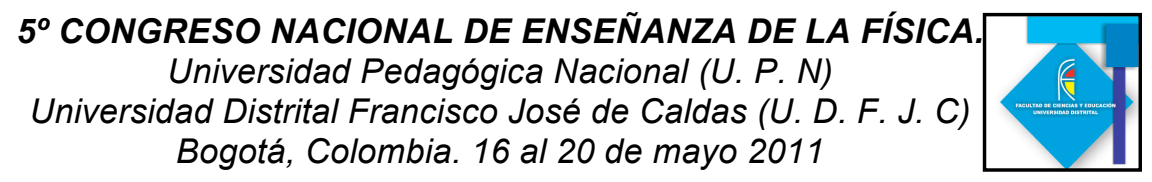

cuenta que sólo eran líneas de pensamiento decidieron crear a Chimi, la pulpa, esta fue la primera cosa consistente del mundo, además hicieron una fusión de semillas germinables, la cual fue dispersada por todo el mundo dando vida a los luceros, soles, planetas, estrellas y finalmente a la vida láctea. Tiempo después crearon la tierra distribuyeron el calor al sol y el frio a la luna, sin embargo todo parecía una pintura, entonces los dioses decidieron darle color a Chimi, transformándolo en Chimini para que este de color e iluminación a la tierra y a su vez cree bosques, ríos y animales. Chimini observó la tierra y se dio cuenta que nada de lo creado tenia vida, ni alegría por lo cual y gracias a la ayuda de la abuela Bague, sufre su última transformación quedando convertido en Chiminigagua, dios supremo, el cual es el encargado de darle vida, movimiento y alegría a todo en la tierra.

Ésta es la cosmovisión muisca que pese al tiempo y los obstáculos que ocasionaron la pérdida de su cultura, sigue presente en la memoria de los abuelos, aquellos que se han encargado de trasmitirla por medio de la tradición oral.

\section{Representación física de la formación del mundo}

La observación de las profundidades del espacio y la necesidad de dar explicación a preguntas tales como: ¿Quiénes somos? ¿De dónde venimos? Y ¿Cómo surgimos? entre otras, han permitido la formulación del modelo cosmológico estándar, llamado Big Bang, que pese a su inconcluso inicio, es el aceptado por la comunidad física perteneciente a la cultura occidental. En este modelo se piensa que el universo tuvo un tamaño nulo, por lo tanto estuvo enormemente caliente lo cual provocó una gran explosión. Un segundo después del Big Bang, la temperatura descendió alrededor de 10.000 millones de grados, en este momento el universo estaba compuesto fundamentalmente por fotones, electrones, neutrinos, sus antipartículas y algunos protones y neutrones. A medida que el universo se expandía su temperatura disminuía, cuando el universo duplica su tamaño la temperatura se reduce a la mitad (Hawking, 1989). Unos segundos después, la temperatura del Big Bang habría descendido a la décima parte de su temperatura inicial provocando una desaceleración de partículas como, los protones y los neutrones, dando inicio a la formación de los primeros núcleos de los átomos. Durante el siguiente millón de años, aproximadamente, el universo continuó expandiéndose. Una vez que la temperatura descendió a miles de grados, los electrones y los núcleos no pudieron vencer la atracción entre ellos, por tanto comenzaron a combinarse formando el átomo. En regiones del universo levemente más densas, la atracción gravitatoria entre las partículas evitó que siguieran expandiéndose, formando nubes de gases, galaxias, estrellas, planetas y todo lo que hoy en día se observa.

\section{Conclusiones}

a) De esta investigación se puede rescatar la importancia de formar maestros etnoeducativos, capaces de mantener viva la cultura de una comunidad en particular.

b) El reconocimiento de otras culturas permite acceder a los miembros de una comunidad al conocimiento de saberes y avances de dichas culturas, los cuales les pueden ayudar a desarrollar habilidades, destrezas y al enriquecimiento en su proceso cultural como grupo. 


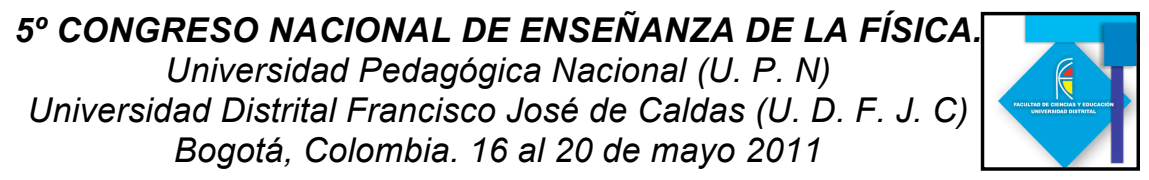

c) El cuento interactivo como estrategia didáctica realza el interés del estudiante por conocer más acerca de un tema, ya que al sentirse involucrado en este, le da la posibilidad de apropiarse de dicho tema.

\section{Referencias bibliográficas}

Carrioni, G. (1992). Formación de indígenas en etnoeducación, Revista Educación y Cultura, 1 (27), 24-27.

Encabo, A. L. (2004). Didáctica de la literatura el cuento, la dramatización y la animación a la literatura. Barcelona: Octaedro-EUB.

Ferris, T. (1998). Informe sobre el universo. Barcelona (España): Drakontos.

Hawking, S.W. (1989). Historia del tiempo del big bang a los agujeros negros. Barcelona: Crítica.

Patiño, G. (1996). Etnoeducación una alternativa cultural transformada, Revista de Ciencias Humanas, 3 (10), 75-82.

Román, M. y Diéz, E. (1994). Currículum y enseñanza, Madrid: EOS

Rozo, J. (1997). Mito y Rito entre los Muiscas. Bogotá: El Buho.

Rozo, J. (1998). Relatos de la antigua Bacatá. Bogotá: Ediciones Naidí. 